\title{
Modelo explicativo del conocimiento organizacional en la Corporación Autónoma Regional del Valle del Cauca - CVC
}

\author{
González Campo, Carlos Hernán* \\ Saltaren Quiñónez, Henry Antonio ** \\ Arenas Quiñones, Nubia Mercedes ***
}

\section{Resumen}

El conocimiento es el activo más importante que posee una organización, en particular para las Autoridades Ambientales, el presente artículo de investigación tiene como objetivo presentar un modelo explicativo del conocimiento organizacional en la Corporación Autónoma Regional del Valle del Cauca - CVC y el diseño de una propuesta para la gestión de conocimiento organizacional, ambos resultantes de la investigación interpretativa basada en el paradigma constructivista utilizados en la metodológica de estudio de caso, en el cual la teoría social no debería basarse únicamente en observaciones empíricas para derivar en leyes generales fundamentales, sino el entender las razones de acción de los actores. Entre los hallazgos más importantes, la investigación revela un cambio institucional, un proceso de transición y de nueva adaptación en respuesta a nuevos objetivos que internamente reflejan el estilo de gobernar y la estructura organizacional y estos a su vez tienen un fuerte impacto en los procesos y en la cultura "cevecina".

Palabras clave: Gestión de conocimiento, generación de conocimiento, transferencia de conocimiento, uso del conocimiento.

Recibido: 10-04-10. Aceptado: 10-09-10

* $\quad$ Autor Principal. Ph.D.(c). Magister en Ciencias de la Organización. Especialista en Mercadeo. Especialista en Gestion del Talento Humano. Economista. Profesor Tiempo Completo Universidad del Valle. E-mail:carlosh@univalle.edu.co. Correspondencia: Universidad del Valle. Sede San Fernando, Edif. 124 Ofic. 1028. Cali - Colombia.

** Msc. Administrador de Empresas. Profesor HC Universidad del Valle. E-mail:hsaltar@univalle.edu.co

*** Msc. Especialista en Sistemas Gerenciales de Ingeniería. Ingeniera Industrial. Consultora Organizacional. E-mail:nmarenasnm@gmail.com 


\title{
Explanatory Model for Organizational Knowledge in the Regional Autonomous Cauca Valley Corporation - CVC
}

\begin{abstract}
Knowledge is the most important asset that an organization possesses, in particular for environmental authorities. The objective of this research is to present an explanatory model for organizational knowledge in the Regional Autonomous Cauca Valley Corporation - CVC and the design for a proposal for managing organizational knowledge, both resulting from interpretive research based on the constructivist paradigm used in case study methodology, in which social theory should be based, not only on empirical observations to derive general, fundamental laws, but rather on understanding reasons for the actors' actions. Among the most significant findings, the research revealed an institutional change, a process of transition and new adaptation in response to new objectives, reflected internally in the style of governance and the organizational structure; these, in turn, have a substantial impact on the Cauca Valley Corporation processes and culture.
\end{abstract}

Key words: Knowledge management, knowledge generation, knowledge transfer, use of knowledge.

\section{Introducción}

El factor humano en las organizaciones ha adquirido una gran relevancia, es normal escuchar del tema en todos los círculos empresariales, académicos, pero, ¿qué es lo que lo hace importante?, ¿en qué consiste su valor para la empresa? La clave está en el conocimiento que tienen los miembros de la organización, y como, a través de éste aportan a su funcionamiento y desarroIlo. En este sentido en una organización es importante la habilidad de sus miembros para aprender unos de otros y contribuir colectivamente a la solución de un problema y/o a la identificación de nuevas oportunidades de negocio.

La Corporación Autónoma Regional del Valle del Cauca (CVC) en Colombia, no es ajena a esta realidad, para cumplir con los fines esenciales que le ha encomendado el Estado Colombiano, re- lacionados con el ejercicio de la autoridad ambiental, la preservación de los recursos naturales y la educación ambiental en el departamento del Valle del Cauca. Necesita generar mecanismos que le permitan estudiar en detalle los recursos y ecosistemas que protege, al igual que las mejores prácticas para transferir el conocimiento que genera entre sus funcionarios, los actores sociales y otras entidades públicas y privadas del País.

A nivel de contextualización, es importante resaltar como la división de la CVC producida a finales del año 1994, como consecuencia de lo dispuesto en la Ley 99 de 1993, fue enfocada principalmente en la creación de la Empresa de Energía del Pacífico EPSA, siendo muy pocos los elementos de gestión y de direccionamiento estratégico para guiar el funcionamiento de la nueva CVC, que continuaría a cargo de las responsabilidades ambientales en el departamento; 
Modelo explicativo del conocimiento organizacional en la Corporación Regional del Valle... González C., Carlos H.; Saltaren Q., Henry A. y Arenas Q., Nubia M.

es así como el diseño de la CVC, aun cuando estuvo acorde con los lineamientos del Departamento Administrativo de la Función Pública, no respondió a las necesidades de la nueva organización. Con conocimiento de las limitantes internas y principalmente por la conciencia del papel de la CVC en la construcción del desarrollo sostenible de la región, a mediados del año 2001, la administración decidió organizar, reorientar y darle foco al quehacer de la CVC, buscando fortalecerla y prepararla para los nuevos retos generados por las responsabilidades de ley, que hasta el momento no habían sido asumidas en toda su dimensión, esfuerzo que aun continúa vigente. El propósito principal, ha sido diseñar una organización que facilite la consolidación de una nueva "Cultura Ambiental en el Valle del Cauca", en donde cada vez sean más los compromisos, las acciones y el número de personas e instituciones que trabajen por el desarrollo sostenible de la región. La obligatoriedad del cumplimiento de disposiciones legales y el reconocimiento del impacto que la gestión de la CVC tiene sobre el desarrollo sostenible del departamento del Valle del Cauca, son el mejor preámbulo para justificar la importancia y necesidad de un modelo de gestión del conocimiento en la CVC.

Los diferentes actores sociales (la comunidad, las empresas, entidades educativas, hospitalarias, gubernamentales, entre otros) están interviniendo y afectando de manera permanente el medio ambiente en el cual desarrollan sus actividades, lo cual obliga a la corporación como ente regulador a fijar las políticas, intervenir en el territorio, generar y transferir conocimientos en materia ambiental a dichos actores sociales, con el fin de prevenir y mitigar la utilización inadecuada de los recursos.

En este contexto, era necesario preguntarse: ¿Cómo está gestionando y cómo debe gestionar el conocimiento organizacional la CVC? Esta pregunta es muy compleja, para su respuesta fue necesario realizar un diagnostico de la organización y plantear un modelo que incluye una propuesta para la gestión del conocimiento en la organización. Con el fin de lograr validez en la investigación empírica, se realizó una encuesta de carácter cuantitativo aplicada al $40 \%$ de los empleados, además se realizaron entrevistas cualitativas a profundidad con personas claves de la organización.

El objetivo general de la investigación fue entonces, identificar la situación existente y diseñar un modelo del conocimiento en la Corporación Autónoma Regional del Valle del Cauca - CVC, el cual explique las dinámicas organizacionales en la generación, la transferencia y su utilización. Algunos de los objetivos específicos fueron: 1) Identificar la percepción de los trabajadores sobre los factores que facilitan la gestión del conocimiento organizacional al igual que las barreras que lo dificultan. 2) Proponer un modelo explicativo de gestión del conocimiento que integre las diferentes variables objeto de estudio, de acuerdo con los resultados del análisis interno, análisis externo, barreras y facilitadores identificados en el diagnostico y soportados por el marco teórico.

La metodología de la investigación en el caso de estudio incluye: el marco teórico, el diagnostico de la situación y tiene como resultado una propuesta de intervención con sus respectivas conclusiones. La investigación fue desarrollada en 
dos etapas: en la primera etapa, una investigación exploratoria a través del análisis de documentos, la observación directa, la aplicación de entrevistas y encuestas a los funcionarios de la CVC, y en la segunda etapa se desarrolla un modelo explicativo de los hallazgos detectados en la primera etapa y una propuesta que busque subsanar las deficiencias encontradas en el diagnostico. Para la aplicación de la entrevista semi-estructurada se realizó una selección de los cargos claves, de acuerdo con su experiencia, conocimiento de la organización y nivel de incidencia sobre las variables objeto de estudio. Finalmente, para el muestreo de las encuestas se aplican los criterios estadísticos más generalizados en el ámbito de la investigación social: Nivel de confianza 95\% y margen de error $5 \%$. Población: 626 personas. Tamaño de la muestra: 249 personas.

\section{Hacia un Modelo de Conocimiento Organizacional (MCO)}

Los autores, luego de realizar una revisión del estado del arte de los modelos de conocimiento organizacional, encuentran que éstos son "islas de discursos" que varían en el nivel de concordancia entre ellos y son construidos desde posiciones filosóficas constructivistas, funcionalistas, estructuralistas o una mezcla de ellas, de acuerdo a la especialidad de cada autor. Los modelos más difundidos como la espiral de conocimiento de Nonaka y Takeuchi (1999), la pirámide del conocimiento de Ackoff (1989), la ecología de la gestión del conocimiento de Snowden (2000), la organización de form-N de Heldund (1994) o el ciclo de vida de McElroy (2003), para nombrar sólo algunos de los más conocidos, se ocupan primordialmente de las características del conocimiento, el proceso del conocimiento, sus componentes, categorías, su definición dentro del ámbito organizacional y la forma de operar en la empresa con el objetivo de lograr una posición estratégica basada en la diferenciación. Son modelos descriptivos que ilustran las relaciones de las variables que cada autor considera fundamentales para la comprensión del fenómeno $y$, algunos de ellos, llegan a describir acciones para su aplicación.

En este contexto, hay que tener en cuenta que algunos recursos de las organizaciones son fáciles de transferir, mientras que el conocimiento, que suele ser tácito, es más difícil de reproducir. Al ser el conocimiento un recurso valioso, e inimitable (Barney, 1991), recurso esencial, y como tal, fuente de ventaja competitiva (Grant, 1996), las empresas buscan la forma más eficiente y efectiva de gestionarlo. Definiendo qué conocimiento la acción de cómo crearlo, transferirlo y utilizarlo. En este sentido, Grant (1996) afirma que cuanto más dinámico es el entorno más sentido tendrá que la empresa base su estrategia en sus recursos y capacidades frente a consideraciones de otro tipo.

A riesgo de ser extremadamente generalista, es posible decir que todos ellos concuerdan con que debe existir un entorno que propicie la creación, adquisición, transferencia y uso del conocimiento al cual hacen referencia como "contexto", "cultura" o "BA". La intención del modelo propuesto, es la de mostrar la importancia de la perspectiva cultural 
Modelo explicativo del conocimiento organizacional en la Corporación Regional del Valle... González C., Carlos H.; Saltaren Q., Henry A. y Arenas Q., Nubia M.

y la de tener un marco de referencia para comprender y diagnosticar como la cultura organizacional ejerce su influencia sobre el conocimiento convirtiéndose así en un facilitador o barrera al mismo. Para alcanzar este objetivo se recurre al rombo filosófico de Renée Bédard (2003), se analiza y adapta la interpretación que hace Stenmark (2001) del conocimiento cultural de Choo (1998) y se supone que cualquier modelo (espiral de conocimiento, la organización Forma-N, etc.) que exprese un proceso en la adquisición, uso y transferencia de conocimiento se suscribe dentro del modelo propuesto sin llegar a negar o afirmar su validez lo que no afectará el análisis realizado.

El modelo propuesto no busca ser una herramienta de gestión, aunque los autores piensan que es posible la identificación de indicadores que permitan medir los resultados de los esfuerzos encaminados a la construcción de una cultura facilitadora de conocimiento como también indicadores que muestren de forma indirecta las mejoras en la creación, adquisición, transferencia, y uso del conocimiento. Pero se reitera que este no es el propósito del modelo, como tampoco lo es de demostrar que puede reproducirse en otras organizaciones. La CVC es una organización que tiene como finalidad para funcionar, la creación, adquisición, transferencia y uso de conocimiento. No lo hace para ser innovadora o crear una ventaja competitiva, el conocimiento es para la CVC la materia prima con que funciona al igual que lo es para la mayoría de los organizaciones que actúan como autoridades ambientales en el territorio Colombiano.

\subsection{Fundamentos conceptuales para un Modelo de Conocimiento Organizacional}

El modelo de conocimiento propuesto se suscribe dentro del marco de referencia constructivista en donde, sin negar que hay una realidad, la visión que se tiene de ella es una construcción mental del individuo que es afectada por el entorno social que la rodea, delimitada por los siguientes principios:

1. Conocer es una capacidad exclusivamente humana que nos permite acceder a nuestras propias representaciones implícitas con la mediación de esos sistemas culturales de representación simbólica y, de esta forma, reconstruirlas (Pozo, 2003). Los seres humanos son (hasta ahora) los únicos capaces de conocer y crear conocimiento.

2. La cultura es parcialmente "recurrente" a su entorno exterior, en otras palabras, conociendo las culturas en donde están inmersas las organizaciones podemos inferir "parcialmente" como es la cultura organizacional.

3. La cultura organizacional es el repositorio del conocimiento organizacional, es amplio, distribuido y dinámico; es el contexto donde el "saber hacer" cobra significado.

4. La acción de conocer involucra el proceso mental de comprender, entender y el aprendizaje que se lleva a cabo en la mente y solo en la mente, sin embargo, gran parte de este proceso involucra interacción con el mundo exterior y el interactuar con otros. Cuando deseamos expresar lo que sabemos, sólo podemos hacerlo 
pronunciando mensajes de una clase u otra -oral, escrito, grafico, gestual o aún 'lenguaje corporal'-. Estos mensajes no transportan 'conocimiento', ellos constituyen 'información', que una mente conocedora puede asimilar, entender, comprender e incorporar dentro de sus propias estructuras de conocimiento. Estas estructuras no son idénticas para la persona que profiere el mensaje y para quien la recibe, porque las estructuras de conocimiento de las personas están, como Schutz (1962) afirma, "determinadas biográficamente". Por tanto, el conocimiento construido a partir de los mensajes nunca puede ser exactamente el mismo que el conocimiento base de donde el mensaje fue emitido (Wilson, 2002).

5. Conocer es mirarnos en el espejo de nuestras representaciones implícitas, haciendo que la carne se convierta en verbo y, a través de esa nueva mirada culturalmente construida, acceder a nuevas formas de conocer el mundo y vivir en él. Todo acto de conocimiento es de algún modo un autorretrato, pero al mismo tiempo es la única forma de superar o reconstruir las restricciones que, como consecuencia de su historia natural, nos impone nuestro sistema cognitivo implícito. Más allá de esas restricciones, otro mundo mental es posible, pero sólo a través del conocimiento, entendido como una reconstrucción cultural de nuestra naturaleza cognitiva más primaria (Pozo, 2003).

6. La cultura organizacional no es un componente más de las organizaciones, es por el contrario un producto de las instituciones (áreas funcionales, departamentos, oficinas especializadas) y los procesos que se llevan a cabo; es el resultado de una estructura social. Además, es simultáneamente actor y acto modificando las instituciones, las estructuras los procesos y así misma.

7. La "gestión del conocimiento" es una alegoría, el conocimiento en sí no se gestiona, podemos observar sus efectos (patentes, procesos, rutinas, productos, información) pero al llevarse éste en el cerebro de cada individuo no es posible "medirlo" directamente, y como principio fundamental en la administración "lo que no se puede medir, no se puede gestionar".

8. Para la creación de un entorno donde el conocimiento fructifique, es necesario contar con: a) las condiciones necesarias, b) los medios adecuados, c) las acciones correctas y d) un liderazgo acertado. Las condiciones necesarias, hace referencia a la cultura organizacional y es resultante de la actuación de las tres últimas: medios, acciones y liderazgo; los cuales a su vez, son el resultado de las instituciones de la organización. Para los autores, este entorno se puede explicar usando las cuatro fuerzas del rombo filosófico de Bédard.

\subsection{Desarrollo de un Modelo de Conocimiento Organizacional}

La cultura corporativa influye en todos y cada uno de los aspectos de la organización (liderazgo, poder, estructuras, procesos, toma de decisiones) pero su relación con el conocimiento organiza- 
Modelo explicativo del conocimiento organizacional en la Corporación Regional del Valle... González C., Carlos H.; Saltaren Q., Henry A. y Arenas Q., Nubia M.

cional es más estrecho y directo. La cultura tal como lo afirman David De Long y Liam Fahey (2000): a) actúa moldeando las suposiciones acerca de lo que es el conocimiento y qué conocimiento vale la pena gestionar, b) define las relaciones entre el conocimiento individual y el conocimiento organizacional, c) crea el contexto para la interacción social que determina cómo el conocimiento será utilizado en situaciones particulares, d) moldea los procesos mediante los cuales el nuevo conocimiento es creado, legitimizado y distribuido en las organizaciones y e) como lo mostró la investigación, determina la forma en que conocimientos obsoletos son abandonados.

Teniendo en cuenta lo anterior, la interpretación que hace Stenmark (2001) del conocimiento cultural de Choo (1998) es incompleta. Tal y como se muestra en la Gráfica 1, la ventana derecha sobre el conocimiento no es una nueva categoría de conocimiento como lo afirma Choo (1998), ni tampoco es un solapamiento entre conocimiento explicito y tácito. Lo que representa son los aspectos en los cuales la cultura se expresa o es visible a través del conocimiento, y la ventana izquierda en la misma gráfica, representa lo que la cultura determina en el conocimiento organizacional. Para representar tanto a la cultura como al conocimiento se recurre a la metáfora del iceberg, planteando que existen paralelismos entre el uno y el otro.

Ambos tienen una zona "visible" producto o resultante de lo que acontece

\section{Gráfica 1. Relación entre Cultura y Conocimiento}

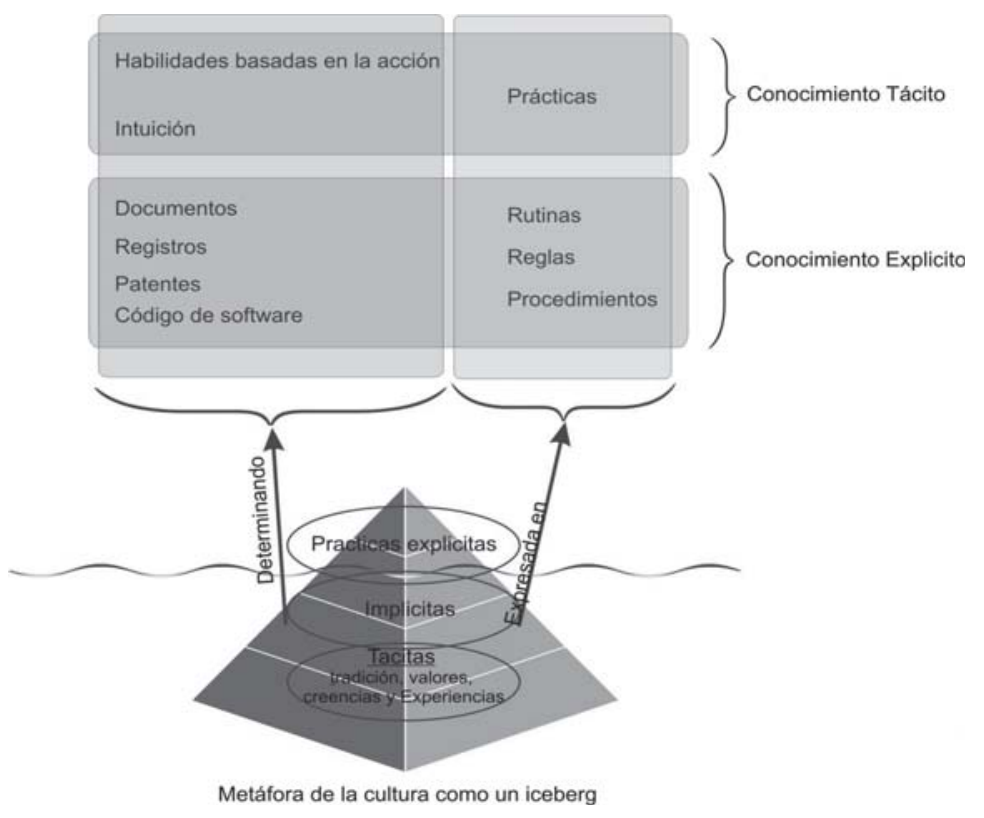

Fuente: Adaptación de los autores de Stenmark, D. (2001:11-14). 
debajo, ambos plantean que existe una zona de muy difícil acceso o imposible de ver y un lugar intermedio donde lo tácito se hace implícito, la línea de flotación. Una diferencia entre los dos es su campo de acción, mientras que el iceberg del conocimiento compete al individuo, el de la cultura hace referencia al grupo social (la organización). De lo que se desprende que la cultura que incorpora todas las normas y reglas no pronunciadas, actúa como un ente de control sobre el individuo y por tanto sobre el conocimiento, teniendo en cuenta que sólo el hombre es capaz de conocer, pero a la vez, la cultura es influenciada por el cúmulo de conocimiento de los individuos.

Siguiendo en este razonamiento y dejando de lado el conocimiento instintivo, aquel que todo ser humano comparte con las demás especies, es posible afirmar que el conocimiento y la cultura son los lados de una misma moneda, pero no en sentido estático de la metáfora, sino más bien un proceso, una dinámica entre fuerzas que se afectan unas a otras de muy variadas maneras siempre en una constante dialéctica y retórica entre ellas. Sin conocimiento no hay cultura y viceversa, puesto que los valores, creencias, tradiciones son en sí mismos conocimientos que dan forma a la cultura y esta a su vez determina los conocimientos que la soportan.

\subsection{El rombo filosófico y la cultura organizacional}

El rombo filosófico de Bédard es un modelo que también usa la metáfora del iceberg tal y como se observa en la Gráfica 2, y se utiliza para traducir un fenómeno particular de una forma figurativa abstracta que pone en evidencia los elementos juzgados esenciales, puede ser una construcción o una representación simplificada que pretende ser operativa, es decir, que puede ser utilizada como una herramienta de observación, de cálculo o de previsión. Puede ser descriptiva, expositiva o explicativa, inductiva o predictiva. Esencialmente es una herramienta de reflexión.

Los cuatro componentes del rombo filosófico aglutinan todas las fuerzas que dinamizan toda actividad en el iceberg del conocimiento organizacional conformando las entradas o los estímulos primarios, por lo tanto se ubican dentro del iceberg de la cultura. Estas cuatro fuerzas interactúan entre sí modificándose y adaptándose, dando forma al entorno antropológico -la cultura organizacional-, y éste último genera fuerzas que a su vez modifican al rombo filosófico de Bédard tal y como se observa en la Gráfica 3.

Aunque se ha utilizado el "estímulo" para hacer referencia a las fuerzas que actúan en la cultura, no se toma en su definición literal, no se espera que a una

\section{Gráfica 2. Rombo filosófico de Renée Bédard}

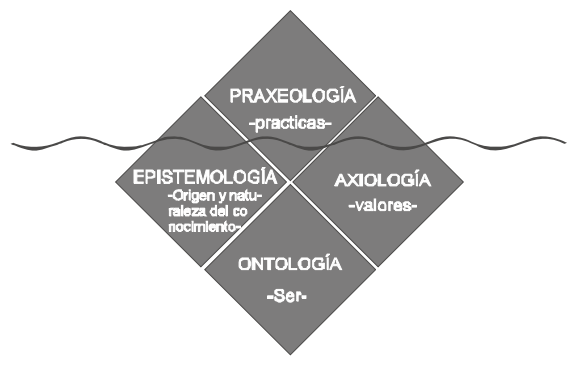

Fuente: Dávila (2003: 91) 
Modelo explicativo del conocimiento organizacional en la Corporación Regional del Valle... González C., Carlos H.; Saltaren Q., Henry A. y Arenas Q., Nubia M.

\section{Gráfica 3. Interacción del rombo filosófico y la cultura organizacional}

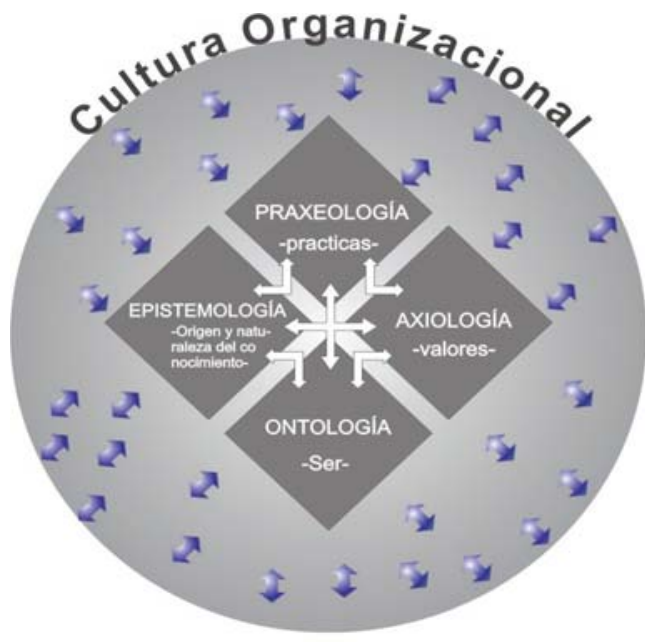

Fuente: Elaboración propia.

estimulo " $x$ " corresponda una reacción " $y$ ", la cultura no es un organismo cibernético, lo que acontece con estas fuerzas es la creación de un conocimiento inicial que no es ni meta-conocimiento (naturaleza y origen del conocimiento), ni conocimiento primario o fundamental, es conocimiento de dirección, de control, de norma y disciplina para la creación, transferencia, uso y reemplazo del conocimiento, cualquiera que sea su categorización, y actúan creando "espacios para el conocimiento" tal y como muestra la Gráfica 4.

Los "espacios para el conocimiento" son atributos, propiedades, condiciones que tiene la organización en sus estructuras, procesos, lugares físicos y mentales que deben ser valorados positivamente para ser considerados como facilitadores del conocimiento.

Espacio de información: Brinda a los miembros de una organización acce- so a la información en diferentes formas como documentos impresos y bases de datos. El acceso a una diversificada y rica colección de información es importante para la creación de conocimiento organizacional puesto que provee riqueza en estímulos y variedad (Nonaka y Takeuchi, 1999). Este espacio de comunicación, es físico, estructural y funcional. Centros de documentación, una intranet funcional y que la mayoría de los empleados pueden utilizarla eficientemente, son ejemplos de espacios de información. Una estructura que facilite el uso de estos espacios y funciones que promuevan su uso crearan una cultura organizacional donde los espacios de información se usen al máximo.

Espacio de comunicación: Permite a los miembros de la organización colectivamente interpretar la información disponible por medio de variados canales de difusión 


\section{Gráfica 4. Creación de Espacios para el conocimiento}

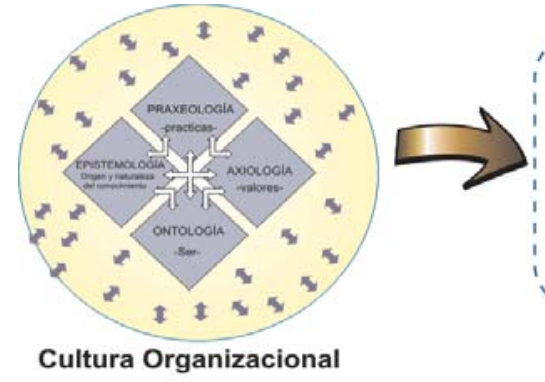

Fuente: Elaboración propia.

y negociación. Estos espacios de comunicación promueven la reflexión permitiendo diferentes puntos de vista e interpretaciones de la información. Las reuniones, los encuentros casuales y conversaciones improvisadas en sitios que favorezcan el intercambio libre de ideas, los foros y los blogs en las intranets, son todos ejemplos de espacios de comunicación.

Espacio de colaboración: Provee los medios para que los miembros de la organización participen y colaboren en actividades de grupos donde compartan la gestión de proyectos y coordinen sus rutinas. En las actividades de colaboración, sus miembros comparten objetivos y manejan un vocabulario común que ayuda a transformar el conocimiento en beneficios para la organización. Los espacios de colaboración, pueden ser grupos interdisciplinarios trabajando en un proyecto común, pueden ser redes de prácticas (alejados unos de otros físicamente) o comunidades de prácticas que tienden puentes de comprensión y aceptación para la consecución de un objetivo común, superando las barreras interculturales que los distancian.
Espacio de Reflexión: Los espacios de reflexión son el lugar donde el conocimiento explicito, implícito y tácito se conectan; corresponde a la línea de flotación en los icebergs. Es donde la información es interiorizada modificando las estructuras mentales del receptor. Este espacio es la construcción en parte de los espacios de información, comunicación y colaboración. Es singular puesto que está en la mente del individuo, pero también está afectado por la epistemología y la axiología.

El modelo propuesto, en la creación de los espacios del conocimiento supone un proceso evolucionista en la organización en la manera que depende profundamente del modo en el cual el presente es residuo del pasado, a través de las experiencias vividas por los procesos de aprendizaje y las rutinas organizativas, sin embargo esta característica evolutiva depende, como todo proceso evolutivo, de la redundancia y del tiempo. La redundancia o procesos y actividades duplicados en las empresas compiten por los recursos limitados para operar y sólo prosperarán aquellos que estén firmemente respaldados por una validez y valoración que se complemente y ratifique en las prácticas, y brinden 
Modelo explicativo del conocimiento organizacional en la Corporación Regional del Valle... González C., Carlos H.; Saltaren Q., Henry A. y Arenas Q., Nubia M.

sentido y razón de ser a la organización y a sus individuos. El factor tiempo limita a que éste proceso evolutivo sólo se den en organizaciones que llevan años operando y han creado una memoria organizacional que es dinámica, capaz de recordar las experiencias positivas y negativas como lecciones aprendidas y "borrar" o "anular" prácticas (conocimientos) que ya no son validos. Finalmente, este proceso evolutivo aun cuando deberá proveer siempre los espacios para el conocimiento, estos cada vez serán más complejos al igual que los procesos de creación adquisición, transferencia y uso del conocimiento.

\subsection{Diagnóstico de la Gestión del Conocimiento en la CVC}

En esta contexto, se han analizado los aspectos más relevantes de la percepción que tienen los funcionarios de la CVC sobre la forma como la organización gestiona el conocimiento, los cuales fueron extractados de las entrevistas realizadas a los informantes clave y de la encuesta aplicada a una muestra de 249 trabajadores de los 626 adscritos a la planta de cargos, correspondientes al tamaño de la muestra para un nivel de de confianza del $95 \%$ y un margen de error $5 \%$. Estos trabajadores fueron seleccionados de la administración central y las 8 direcciones regionales proporcionalmente al tamaño de la población que les corresponde. Para cada variable independiente (generación, transferencia y uso del conocimiento) fue establecida la correlación con las variables dependientes (cultura organizativa, estilo directivo, aprendizaje, tecnologías de información, estructuras y procesos), detectando los aspectos contextuales que actúan como facilitadores o inhibidores, resultantes del análisis de las entrevistas y encuestas. A continuación se presentan los aspectos más relevantes, en cuanto a la generación, transferencia y uso del conocimiento en la organización objeto de estudio.

A nivel de la generación del conocimiento, la CVC cuenta con mecanismos, procesos, metodologías, herramientas, equipos y personal competente para generar nuevo conocimiento, este conocimiento es el producto generalmente planificado de investigaciones realizadas en los laboratorios, centros de investigación o el trabajo de campo en el territorio; la organización también posee tecnología apropiada que permite generar información valiosa y confiable para la toma de decisiones y la planificación; con excepción de algunas temáticas ambientales donde no existe la suficiente información ni los planes de manejo para cada una de estas áreas.

Estos facilitadores son afectados por algunos elementos que actúan como barreras, como es el caso de la cultura corporativa, pues no propicia suficiente o correctamente las practicas que ayuden al grupo y sus individuos a ser creativos e innovadores; hay carencia pero no absoluta de mecanismos que identifiquen el nuevo conocimiento, incentivos que lo alienten y espacios que lo faciliten; las directivas de la institución muestran un compromiso en crear estructuras y actividades para promover la creación de conocimiento pero los funcionarios lo valoran como insuficiente o poco exitosa en el logro de los objetivos. En cuanto al cumplimiento de políticas que promuevan el aprendizaje, la percepción de los funcio- 
narios es que no hay apoyo para que ellos aprendan, y aquí hay que resaltar que hace referencia a la práctica de aprender haciendo y no a la formación cognoscitiva adquirida en las universidades o en la capacitación externa. Esta conclusión es muy debatible para una organización cuyo lema es y ha sido "aprender haciendo" desde su creación misma.

Algunos de los entrevistados piensan que hay que conocer que es lo que está pasando en el territorio en términos del medio ambiente, debería plantearse una metodología científica y sistémica para determinar las necesidades que apunten al cumplimiento de la misión o razón de ser de la Corporación; la principal barrera es la falta de una estructura del conocimiento que organice e integre las funciones del día a día con las metas propuestas y permita conocer que procesos optimizan el aprendizaje y su implementación posterior.

En cuanto a la información que posee cada persona, la ley general de archivos obliga a los funcionarios próximos a jubilarse a realizar una entrega de documentos al archivo, pero no existe una política formal y un proceso que apoye la generación y transferencia de conocimiento entre individuos. La CVC, cuenta hoy por hoy, con un gran desarrollo en tecnologías de información y comunicación (hardware, redes, datos y software), esta infraestructura es un facilitador y punto de partida para la generación de conocimiento, lo mismo que el inicio de la estandarización de los procesos; sin embargo, la falta de comunicación entre los diferentes funcionarios y la incompatibilidad (parcial) entre los sistemas de informa- ción y los procesos, actúan más como barrera que como facilitador.

Otra barrera identificada es la especialización o dominio de temas específicos por parte de unos pocos funcionarios, los cuales no muestran interés de adquirir nuevo conocimiento o en compartir abiertamente su saber, quizás por temor a que esto pueda reducir su estatus o poner en peligro su empleo. Igualmente consideran una barrera la falta de relevo generacional que ocasiona la pérdida de la formación del personal que tiene mayor antigüedad y están cerca de la jubilación o ya está jubilado, puesto que la CVC ha tenido una tradición oral en la transferencia de conocimiento y no es fuerte el proceso de documentación sobre todo la sistematizada. Otra de las causas de un relevo generacional deficiente, obedece a la disminución del número de empleados, ocasionado por la necesidad de reorganizar las cosas y decidir que abarcar y que no, sin tener en cuenta en la conformación de los grupos de trabajo una composición adecuada de funcionarios nuevos y antiguos que faciliten la transferencia de experiencias.

Mientras al analizar la transferencia de conocimiento, el nuevo valor que adquiere en la cultura "cevecina" la gestión del día a día, el estar siempre ocupado haciendo seguimiento de proyectos y demás actividades aparece como una barrera en la transferencia del conocimiento, está reemplazando el valor que anteriormente tenía el tomar tiempo para formarse y aprender aunque la necesidad y el interés por aprender sigue siendo parte muy relevante en la cultura. Las iniciativas de transferencia del conocimiento tienden a tener pocos resultados, por 
Modelo explicativo del conocimiento organizacional en la Corporación Regional del Valle... González C., Carlos H.; Saltaren Q., Henry A. y Arenas Q., Nubia M.

ejemplo la experiencia con la práctica del "viernes ambiental" espacio creado para reunir a las personas para que contaran sus metodologías y experiencias no dio frutos en términos generales por la falta de tiempo para asistir. En gran parte la falta del tiempo dificulta el contacto y la interacción entre las fuentes de conocimientos y los receptores, según palabras textuales de un entrevistado siempre están todos muy ocupados, 'siempre están súper programados', con gran cantidad de reuniones que no les permiten formarse mejor. Otro obstáculo para la comunicación del conocimiento hace énfasis en el personal antiguo que en ocasiones es renuente a recibir nuevo conocimiento, con la creencia de que lo saben todo, pero también en la negación de transferir su propio conocimiento a terceros.

Los entrevistados consideran que el mecanismo más eficaz para transferir el conocimiento consiste en colocar al lado de las personas que tienen el conocimiento, las personas nuevas, los cuales en la cotidianidad aprenderán de los más antiguos, este mecanismo era el que anteriormente usaba la CVC. Lave y Wenger (1991) proponen un proceso llamado Legitimación de la participación Periférica (Legitimate Peripheral Participation (LPP) por sus siglas en ingles) en las comunidades de practica (CoPs), las cuales sustentan la transferencia y creación de conocimiento. La socialización en las comunidades de prácticas se da simultáneamente con instrucción y la demostración. Vemos entonces que, aunque el estilo directivo propende por la creación de mecanismos de transferencia de conocimiento a través de procesos (poner a antiguos y nuevos a trabajar juntos) hace falta que haya una participación activa; pero para esto es necesario hacer cambios en la cultura organizativa, específicamente en las subculturas.

Para el almacenamiento y transferencia del conocimiento, la mayoría de los entrevistados concordaron que como herramienta que apoya estas actividades cuentan recientemente con lo último en tecnologías de información los cuales soportan varios sistemas de información como el SIPA (el sistema de información ambiental), pero hay que resaltar la palabra recientemente, antes de ello, en los últimos 20 años, mucho del conocimiento generado, no fue conservado. Las bases de datos, la intranet, las páginas web es un mecanismo eficaz para transferir conocimiento, ellos poseen información de todas las áreas e índices de contenido de material impreso para realizar búsquedas con mayor agilidad, sin embargo el medio más usado para difundir la información (no el conocimiento) es a través del documento escrito, el cual está disponible en el centro de Documentación, tanto para el personal interno como para el externo. Muchas veces el documento es multimedia (video, sonido, entre otros).

En cuanto al uso del conocimiento, para algunos entrevistados, en la corporación hay conocimientos muy claros en temáticas especificas, pero se requiere mejorar la articulación que debe existir entre los profesionales de diferentes especialidades, pues partamos de que el medio ambiente es uno solo, y cualquier acción que se emprenda sobre un recurso natural puede afectar a otro; sin embargo, se reconoce el esfuerzo y compromiso de la alta dirección, pues brinda oportunidades para aplicar el conoci- 
miento adquirido, aunque existen barreras culturales como las ya citadas en ítems anteriores, que hacen que no se potencialice este elemento facilitador.

Existen brechas entre el conocimiento adquirido con la formación, y el conocimiento realmente necesario, esto sucede porque los campos de aplicación no siempre son desarrollados, por ejemplo si los funcionarios asisten a seminarios que tienen que ver con el intercambio climático, pero no logran que la corporación implemente acciones concretas referente a este tema, entonces la corporación posiblemente está en una dinámica tal que no le da cabida a algunos conocimientos que poseen las personas por motivación propia o capacitación; pero también en algunos casos los mismos empleados no hacen el intento de dar una aplicación concreta a sus conocimientos. En este sentido, la Corporación (conformada por todos sus empleados) deberá reconsiderar que debe conocer que sea congruente con lo que debe hacer.

\section{Propuesta de un Modelo de Conocimiento Organizacional para la CVC}

Después de determinar el estado actual del conocimiento en la Corporación Autónoma Regional del Valle del Cauca CVC, es de gran valor agregado presentar una propuesta que articule las diferentes variables objeto de estudio y constituya una alternativa válida para dar respuesta a las falencias detectadas en el diagnostico. Esta propuesta está fundamentada en los preceptos que soportan el modelo explicativo y tiene como propósito orientar a la or- ganización en el desarrollo de actividades planificadas que contribuyan a la generación de los espacios para el conocimiento (espacio de información, espacio de comunicación, espacio de colaboración y espacio de reflexión). En los cuadros que se presentan a continuación, para cada variable independiente se presenta los objetivos y estrategias sugeridas.

En el proceso de creación del conocimiento es de vital importancia estimular la interacción entre los individuos, en este orden de ideas es importante recordar que según Grant (2000), la generación del conocimiento contiene las siguientes actividades: a) La creación interna de conocimiento que se obtiene de la investigación y diseño dentro de la empresa; b) el aprendizaje por acción a través del entrenamiento en el trabajo, experimentos y simulaciones y c) la adquisición y el acceso del conocimiento externo mediante licencias, subcontrataciones, alianzas estratégicas, proveedores, clientes, universidades, laboratorios de investigación, inventores independientes, agencias gubernamentales, implementación de tecnología y de sistemas de información.

Adoptando esta clasificación pero sin perder de vista el contexto de la organización, se sintetiza a continuación, la propuesta (Cuadro 1).

Cuando se habla de transferencia del conocimiento se hace referencia al conocimiento que es comunicado desde un agente a otro. Este proceso tiene lugar a su vez entre individuos o entre grupos y la organización en general (Camelo, 2000). Es en este sentido en el que se estructuran los objetivos que se presentan a continuación (Cuadro 2). 
Modelo explicativo del conocimiento organizacional en la Corporación Regional del Valle... González C., Carlos H.; Saltaren Q., Henry A. y Arenas Q., Nubia M.

\section{Cuadro 1. Propuesta relacionada con la Generación del Conocimiento Organizacional en la CVC}

\begin{tabular}{|c|c|}
\hline Objetivo & Estrategias \\
\hline $\begin{array}{l}\text { Concientizar a los funcionarios nuevos } \\
\text { y a los vinculados a la planta de perso- } \\
\text { nal sobre la importancia del conoci- } \\
\text { miento como elemento fundamental } \\
\text { para el logro de los fines Corporativos. }\end{array}$ & $\begin{array}{l}\text { - Definir mecanismos para incentivar la generación y } \\
\text { adopción de nuevas ideas. } \\
\text { - Fomentar la participación activa de los Directivos y } \\
\text { Coordinadores en el proceso. } \\
\text { - Estructurar y formalizar un programa de inducción - } \\
\text { re-inducción institucional, que incluya el reconoci- } \\
\text { miento del territorio. } \\
\text { - Incluir funcionarios con amplia experiencia en la Cor- } \\
\text { poración y con conocimiento del territorio en los pro- } \\
\text { gramas de inducción - re-inducción. }\end{array}$ \\
\hline $\begin{array}{l}\text { Generar un conocimiento integral de los } \\
\text { recursos Naturales del Valle del Cauca. }\end{array}$ & $\begin{array}{l}\text { - Generar directrices orientadas a definir las temáticas } \\
\text { ambientales sobre las cuales debe desarrollar la Cor- } \\
\text { poración su Modelo de Conocimiento Organizacional. } \\
\text { - Crear Círculos de Intercambio de Conocimiento en el } \\
\text { cual se discutan, analicen, concluyan y generen pro- } \\
\text { puestas relacionadas con la conservación del medio } \\
\text { ambiente y los recursos naturales. }\end{array}$ \\
\hline $\begin{array}{l}\text { Orientar los esfuerzos de capacitación } \\
\text { hacia el desarrollo de competencias re- } \\
\text { queridas por la organización. }\end{array}$ & $\begin{array}{l}\text { - Introducir como entradas principales al plan de forma- } \\
\text { ción los resultados de la evaluación de competencias, } \\
\text { los productos (conocimiento) generados por los centros } \\
\text { de competencias y las temáticas ambientales prioriza- } \\
\text { das, a fin de garantizar que la inversión en formación de } \\
\text { respuesta a las necesidades de la Corporación. }\end{array}$ \\
\hline
\end{tabular}

Fuente: Elaboración Propia.

Esta variable dependiente de uso del conocimiento, fue introducida como resultado de la revisión bibliográfica, donde se encontró la reincidencia de diferentes autores, donde la incluyen como un elemento fundamental de la gestión del conocimiento organizacional. Se entiende la utilización del conocimiento como el conjunto de actividades relacionadas con la aplicación del conocimiento a los procesos del negocio para adaptarse mejor y más rápido a los cambios del entorno y transformar continuamente la resultante de la utilización del mismo en beneficios para la organiza- ción y la comunidad afectada, mediante el desarrollo de unos procesos, infraestructuras y cultura adecuados, desde el nivel estratégico hasta el operativo; bajo este contexto se presenta a continuación la propuesta (Cuadro 3).

De acuerdo con los resultados de la investigación, se puede concluir que es viable la implementación de un Modelo de Conocimiento Organizacional en la Corporación Autónoma Regional del Valle del Cauca-CVC-, pues esta cuenta con personal calificado ( $48 \%$ de la planta de personal está conformada por profesionales, de los cuales el $36 \%$ tienen forma- 


\section{Cuadro 2. Propuesta relacionada con la Transferencia del Conocimiento Organizacional en la CVC}

\begin{tabular}{|c|c|}
\hline Objetivo & Estrategias \\
\hline $\begin{array}{l}\text { Fomentar la interacción y comunicación } \\
\text { de los trabajadores en torno a la transfe- } \\
\text { rencia del conocimiento. }\end{array}$ & $\begin{array}{l}\text { - Estructurar y formalizar un programa de Tutores } \\
\text { donde los funcionarios de mayor experiencia con- } \\
\text { formen equipos de trabajo con los funcionarios } \\
\text { nuevos, con el fin de brindar un correcto relevo ge- } \\
\text { neracional. } \\
\text { - Fomentar los intercambios de experiencias entre pa- } \\
\text { res (con una fluidez del conocimiento horizontal). } \\
\text { - Abrir espacios de socialización e intercambio de } \\
\text { conocimiento relevante para la organización, } \\
\text { orientado a los grupos de interés. } \\
\text { - Establecer convenios de Cooperación que permi- } \\
\text { tan aumentar la transferencia del conocimiento ín- } \\
\text { terganizacional. }\end{array}$ \\
\hline $\begin{array}{l}\text { Optimizar el uso de los sistemas de infor- } \\
\text { mación como soporte de los procesos de } \\
\text { transferencia del conocimiento. }\end{array}$ & $\begin{array}{l}\text { - Crear la biblioteca virtual alimentada con los pro- } \\
\text { ductos de la investigación de la Corporación y de } \\
\text { los convenios de cooperación interinstitucional. } \\
\text { - Inventariar el conocimiento formalizado por escrito } \\
\text { en publicaciones, informes y otros documentos a } \\
\text { fin de conocer la información disponible. } \\
\text { - Definir políticas y lineamientos para la actualización } \\
\text { de las bases de datos de conocimiento ambiental y la } \\
\text { alimentación del Sistema de Información. }\end{array}$ \\
\hline
\end{tabular}

Fuente: Elaboración Propia.

\section{Cuadro 3. Propuesta relacionada con la Utilización del Conocimiento Organizacional en la CVC}

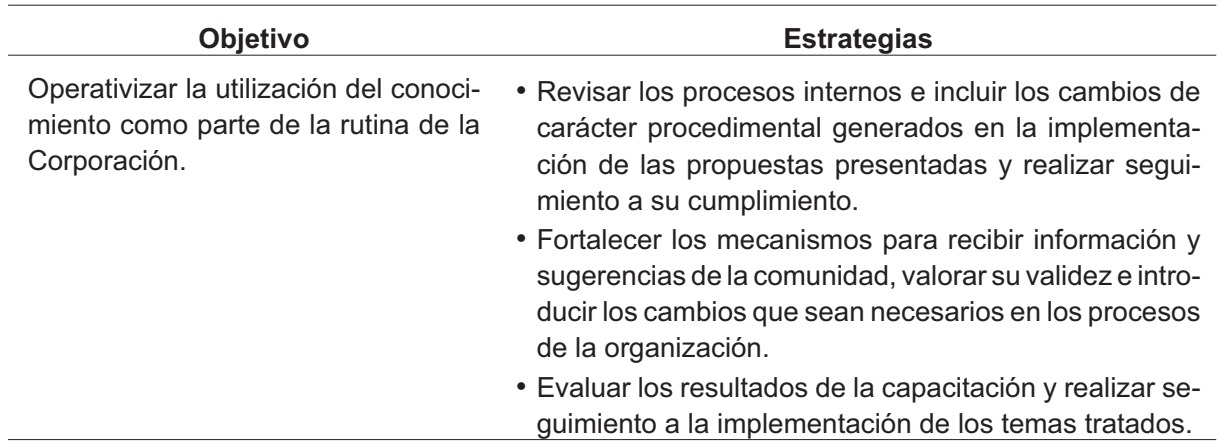

Fuente: Elaboración Propia. 
Modelo explicativo del conocimiento organizacional en la Corporación Regional del Valle... González C., Carlos H.; Saltaren Q., Henry A. y Arenas Q., Nubia M.

ción avanzada y el $23 \%$ tienen formación técnica o tecnológica), recursos financieros, tecnológicos y una tradición del aprender haciendo, que pueden facilitar el proceso, pero para ello es necesario fortalecer el Compromiso y los conceptos del Nivel Directivo relacionados con el tema; concientizar a las áreas de apoyo sobre cómo sus actuaciones pueden fortalecer o debilitar el cumplimiento de los fines esenciales de la organización y articular la gestión de las Direcciones Ambientales Regionales a las actividades de generación y transferencia del conocimiento, pues es en éstas dependencias donde hay un contacto real con las comunidades y los ecosistemas que están siendo afectados.

Es igualmente importante generar los espacios y mecanismos que incentiven la participación del personal en la generación de ideas de mejoramiento y posterior implementación, a partir de la expedición de políticas claras de incentivos que motiven a los funcionarios a compartir el conocimiento que poseen y a materializarlo en acciones prácticas y apoyar la creación de los círculos intercambio que permita ayudar a otros funcionarios a encontrar razones de interés personal que justifique compartir sus conocimientos persiguiendo una meta común. Dentro de la construcción de dichos espacios, también se debe trabajar en la creación de bases para el desarrollo de una red de interacción entre la comunidad, contratistas, personal jubilado o retirado, entidades educativas y ambientales tanto a nivel nacional como internacional, que facilite el contacto o relaciones de los funcionarios con otras comunidades de práctica o investigadores en materia ambiental.
Finalmente, sin ser menos importante se recomienda a la CVC que tenga en cuenta al movilizar los trabajadores entre las diferentes áreas de la organización, que exista una distribución más o menos equitativa entre contratistas, personal de planta joven y personal de planta próximo a jubilarse, a fin de garantizar que en la práctica de las actividades diarias se dé de manera natural el relevo generacional, facilitando la transmisión de experiencias y conocimientos a través de la ejecución de procesos y proyectos en conjunto.

\section{Conclusiones}

Entre los hallazgos más importante de la investigación obtenidos a través del análisis de las encuestas, entrevistas, análisis interno-externo, se revela una Corporación que en términos generales está en "punto medio" en cuanto a la gestión del conocimiento organizacional. Pero este "punto medio" no significa que esta todo a medio hacer, más bien refleja un cambio institucional, un proceso de transición y de nueva adaptación en respuesta a nuevos objetivos que internamente reflejan el estilo de gobernar y la estructura organizacional y estos a su vez tienen un fuerte impacto en los procesos y especialmente en la cultura "cevecina".

El modelo explicativo propuesto puede extenderse a otras corporaciones autónomas regionales ambientales de cualquier país. Teniendo en cuenta que sus objetivos son comunes, sus características serán similares entre ellas. Características que soportan la premisa establecida en el modelo explicativo, el cual afirma que la cultura es parcialmente "re- 
currente" a su entorno exterior, es decir, conociendo la cultura en donde está inmersa la organización, podemos inferir "parcialmente" como es la cultura de dicha organización.

De las variables analizadas, la cultura organizacional es la que tiene mayor influencia sobre el conocimiento organizacional. Condicionando de manera directa los resultados de las otras variables; esta afirmación la podemos soportar con los planteamientos de David W. De Long y Liam Fahey en el 2000, quienes afirman que la cultura incorpora todas las normas, o reglas, no pronunciadas acerca de cómo se debe distribuir el conocimiento entre la organización y los individuos dentro de ella; definiendo el conocimiento que pertenece a la organización y conocimiento que permanece bajo el control de los individuos y las subunidades.

La investigación confirma que la Cultura Organizacional moldea las percepciones y los comportamientos; una manera en que lo hace es al establecer el contexto organizacional para la interacción social. La cultura de una empresa, y las relaciones entre sus subculturas, ampliamente moldean cómo el nuevo conocimiento acerca del medio externo es creado, legitimizado (o rechazado) y distribuido a través de una organización.

El abandono o retiro de sus cargos, de los individuos con gran experiencia, sin un proceso previo de transferencia del conocimiento, ocasiona una gran pérdida del "saber-hacer" de la institución y en una gran barrera para el acceso al conocimiento y al aprendizaje para los empleados que los sustituyen. Cuando hablamos de la rotura de conocimiento en la organización, hace referencia a la pérdida o fugas del conocimiento causado por la extensa problemática de la pérdida del personal importante en la organización. Esta fuga puede ser en dos formas, la primera forma es en las prácticas, referidas como rutinas de la organización en el uso del conocimiento y que frecuentemente tienen un componente tácito incrustado en parte en las destrezas de los individuos y otra parte en los arreglos sociales para colaborar (Nelson y Winter, 1982); la segunda forma la encontramos cuando un empresa contratista encargada de llevar a cabo una función -en muchos casos misional de la Corporación- deja de prestar sus servicios a la firma, en esta situación la pérdida de conocimiento es total si antes el conocimiento no ha sido estructurado de forma explícita en información, esto es, documentos, instructivos, planos, programas de computadoras.

\section{Referencias bibliográficas}

Ackoff, Russell Lincoln (1989). From Data to Wisdom. Journal of Applied Systems Analysis, 16, pp. 3-9.

Bédard, Renee (2003). Los Fundamentos del Pensamiento y las Prácticas Administrativas. AD-MINISTER, 3, pp. 68-88.

Camelo Ordaz, Carmen (2000). La Estrategia de la Diversificación Interna: Una aproximación desde la Teoría basada en el Conocimiento. Madrid. Biblioteca Civitas Economía y Empresa.

Choo, Chun Wei (1998). The Knowing Organization: How Organizations use Information to Construct Meaning, Create Knowledge and Make Decisions. Oxford, Oxford University Press.

De Long, David y Fahey, Liam (2000). Diagnosing cultural barriers to knowledge 
Modelo explicativo del conocimiento organizacional en la Corporación Regional del Valle... González C., Carlos H.; Saltaren Q., Henry A. y Arenas Q., Nubia M.

management. Academy of Management Executive. 14(4), pp. 113-27.

Grant, Robert (1996). Towards a knowledge-based theory of the firm. Strategic Management Journal, 17(10), pp.109-122.

Grant, Robert (2000). Shifts in the world economy: the drivers of knowledge management. En C. Despres y D. Chauvel (eds). Knowledge Horizonts: the present and the promise of knowledge management. Butterworth-Heinemann. Massachussets. $1^{\mathrm{a}}$. Edición.

Grant, Robert (2000). Dirección estratégica: conceptos, técnicas y aplicaciones. Civitas, Madrid. $4^{\text {a }}$ Edición).

Kotabe, Masaaki, Dunlap-Hinkler, Denise, $\mathrm{Pa}$ rente, Ronaldo, and Mishra, Harsh (2007) Determinants of Cross-National Knowledge Transfer and Its Effect on Firm Innovation. Journal of International Business Studies, 38(2), pp. 259-282.

Lave, Jean y Wenger, Etienne (1993). Situated learning: legitimate peripheral participation. Cambridge Univesity Press. Nueva York.

McElroy, Mark (2003) The new knowledge management: complexity, learning, and sustainable innovation. Butterworth Heinemann. New York.
Nelson, Richard y Winter, Sidney (1982). An evolutionary theory of economic change. Cambridge, MA: Belknap Press.

Nonaka, Ikujiro y Takeuchi, Hirotaka (1999). La organización creadora de conocimiento. Oxford University Press. México.

Pozo Municio, Juan Ignacio (2003). Adquisición de conocimiento: cuando la carne se hace verbo. Morata. España.

Snowden, David (2000). Organic knowledge management Part one. Knowledge Management. pp.14-17.

Stenmark, Dick (2001). The Relationship between Information and Knowledge. Proceedings of IRIS 24. Ulvik. Norway. pp. 11-14.

Takeishi, Akira (2001). Bridging Inter- and Intra-Firm Boundaries: Management of Supplier Involvement in Automobile Product Development. Strategic Management Journal, 22, pp.403-433.

Small, Cynthia y Sage, Andrew (2005). Knowledge management and knowledge sharing: A review. Information Knowledge Systems Management, 5, pp.153-169. 